\title{
Improvement of photoluminescence intensity and film morphology of perovskite by lonic liquids additive
}

\author{
Dongyang Shen ${ }^{1}$, Chengzhao Luo ${ }^{1}$ Ronghong Zheng ${ }^{1}$, Qinyi $\mathrm{Li}^{1}$, and Yu Chen ${ }^{1,2, *}$ \\ ${ }^{1}$ School of Optoelectronic Science and Engineering, Soochow University, Suzhou 215006, P. R. China \\ ${ }^{2}$ National University of Singapore Suzhou Research Institute Dushu Lake Science and Education Innovation District Suzhou 215123, P. \\ R. China
}

\begin{abstract}
Metal halide perovskites have received much attention for their application in light-emitting diodes (LEDs) and solar cells in the past several years. Among them, 2D and quasi-2D perovskite with organic long-chain cations introduced have drawn significant attention. However, while improving wet and thermal stability, as the grain size becomes smaller, more defects introduced at the grain boundary and surface, resulting in the increase of non-radiative recombination is becoming the main problem which should be faced by 2D/quasi-2D perovskite materials. Here, we report a new strategy employing ionic liquid named 1-Ethyl-3-methylimidazolium bis(trifluoromethylsulfonyl)imide(EMB).By adding a small amount of ionic liquid to the precursor, the defect was effectively passivated and the photoluminescence intensity was increased by 11 times and the fluorescent lifetime was increased by about 1.5 times. The flatness of the prepared perovskite thin films has also been effectively improved.
\end{abstract}

\section{Introduction}

Due to the advantages of high luminous efficiency, high quantum yield, pure color and adjustable band gap, metal halide perovskites has attracted great attention of researchers recently ${ }^{[1-9]}$. In many perovskite families, the two/quasi-two-dimensional perovskite structures formed by introducing organic long-chain cations to cut threedimensional bulk phase are attracting more and more attention. The chemical formula of quasi-2D perovskite is demonstrated as $L_{2} A_{n-1} B_{n} X 3_{n+1}$, where $L$ represents large-size organic spacer cations, such as phenylethylamine (PEA) and butylamine (BA), A represents monovalent cation $\left(\mathrm{Cs}^{+} 、 \mathrm{MA}\left(\mathrm{CH}_{3} \mathrm{NH}_{3}{ }^{+}\right)\right.$、 $\mathrm{FA}\left(\mathrm{HC}\left(\mathrm{NH}_{2}\right)_{2}{ }^{+}\right.$etc.) . M represents divalent cation $\left(\mathrm{Pb}^{2+} 、 \mathrm{Sn}^{2+}\right.$ etc.). And the $\mathrm{X}$ represents halogen anions $\left(\mathrm{I}^{-}, \mathrm{Br}^{-}, \mathrm{Cl}^{-}\right.$etc.). The introduction of organic long-chain cations improves the hygrothermal stability of quasi-two-dimensional perovskite. Morever, organic long-chain cations is possible to confine the structural freedom, stabilize the oriented structure, and favor to form 2D perovskite film with better crystallinity and orientation $^{[10]}$.

Although the stability has been improved to a certain extent, converting three-dimensional into quasi-twodimensional perovskite introduces more defects on the surface or at the grain boundaries due to the reduction of crystal sizes $^{[11]}$. The increase of defects will further make it easier for excitons to be trapped by defects and then form non-radiative recombination. Some of the most noteworthy approaches include doping the perovskite materials with alkalications, which has been demonstrated to control bulk defects and eliminate hysteresis ${ }^{[12-14]}$. That can greatly improve the quality of perovskite morphology and reduce the density of trap states. Another largely explored method is the introduction of additives into the perovskite precursor solution prior to layer deposition. Ionic liquid can be used as an additive to greatly improve the quality of perovskite morphology and reduce the density of trap states in the quasi-two-dimensional material.

In this study, we report a new defect passivation strategy employing a novel ionic liquid, 1-Ethyl-3methylimidazolium bis(trifluoromethylsulfonyl) imide (EMB). By adding a small amount of EMB to the precursor, the photoluminescence intensity, fluorescent lifetime, and perovskite film quality were significantly improved. The maximum light intensity of the perovskite film was increased by 10 times, and the fluorescent lifetime was increased by about 1.5 times. The prepared perovskite film obtains the flatness of root mean square as low as 3.27.

\section{Materials and Methods}

\subsection{Materials}

Phenylethylammonium bromide(PEABr,99.999\%), lead bromide (PbBr2,99.999\%),methylammonium bromide (MABr,99.999\%) were purchased from Xi'an Polymer Light Technology Corp. dimethyl sulfoxide (DMSO,>99.9\%) was purchased from Aladdin. All

\footnotetext{
* Corresponding author: chenyu_ny@suda.edu.cn
} 
solvents and materials were used directly without further purification.

\subsection{Methods}

$\mathrm{PEABr}, \mathrm{MABr}$, and $\mathrm{PbBr}_{2}$ were dissolved in anhydrous DMSO at a molar ratio of 2:2:3 to form a $0.5 \mathrm{M}$ precursor solution. The mixture was stirred overnight and filtered through a $0.45 \mu \mathrm{m}$ polytetrafluoroethylene membrane before using. The substrate was ultrasonically treated with detergent, deionized water, acetone and isopropanol for $15 \mathrm{~min}$ in sequence, and then dried with nitrogen flow. After treatment with ultraviolet (UV)-ozone plasma for 15 minutes, The substrate was transferred to a glove filled with nitrogen $\left(\mathrm{H}_{2} \mathrm{O}<0.1 \mathrm{ppm}, \mathrm{O}_{2}<0.1\right.$ ppm). $100 \mu \mathrm{L}$ precursor was dropped to spin coating with speed at $5000 \mathrm{rpm}$ for $60 \mathrm{~s}$ and baked at $100{ }^{\circ} \mathrm{C}$ for 10 min. $200 \mu \mathrm{L}$ chlorobenzene was dropped as antisolvent at 30 s.

Table 1 The molar ratio of $\mathrm{PEA}_{2} \mathrm{MA}_{2} \mathrm{~Pb}_{3} \mathrm{Br}_{10}$

\begin{tabular}{|c|c|c|c|c|c|}
\hline & $\begin{array}{c}\text { PEABr(mm } \\
\text { ol) }\end{array}$ & $\operatorname{MABr}(\mathbf{m m o l})$ & $\operatorname{PbBr}_{2}(\mathbf{m m o l})$ & DMSO(mL) & EMB(mmol) \\
\hline w/o & 0.33 & 0.33 & 0.5 & 0.25 & 0 \\
\hline $5 \mathrm{wt} \%$ & 0.33 & 0.33 & 0.5 & 0.25 & 0.025 \\
\hline $10 \mathrm{wt} \%$ & 0.33 & 0.33 & 0.5 & 0.25 & 0.05 \\
\hline $15 \mathrm{wt} \%$ & 0.33 & 0.33 & 0.5 & 0.25 & 0.075 \\
\hline
\end{tabular}

\section{Results and Discussion}

A one-step solvent exchange method was used to prepare $\mathrm{PEA}_{2} \mathrm{MA}_{2} \mathrm{~Pb}_{3} \mathrm{Br}_{10}$ perovskite films. The image of perovskite films is shown in Figure 1a, we can see the obvious difference in photoluminescence intensity under ultraviolet excitation (365 nm). We performed steadystate photoluminescence (PL) measurement for the prepared perovskite film, and the results are shown in Figure 1b. Compared with the film without EMB added, the PL intensity was significantly increased by about 911 times when ionic liquids was introduced in different ratio.Among them, $5 \mathrm{wt} \%$ EMB added has a maximum PL intensity, approximately 11 times than the initial. The emission peak of the pristine perovskite film was located at around $520 \mathrm{~nm}$. while the PL peak of the perovskite films with ionic liquids-treated located at about $514 \mathrm{~nm}$ was slightly blue-shifted. a)
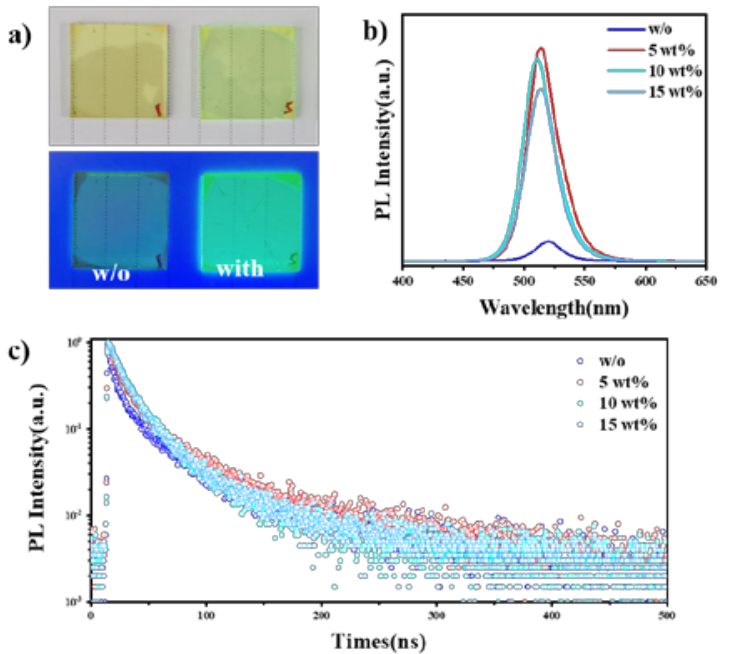

Figure 1. The images and PL characteristics of quasi-2D perovskite films. a) The emission images of perovskite films with and without ionic liquids treatment under ultraviolet excitation (365 nm). b) Steady PL spectra.c) time-resolved photoluminescence spectra.

It could be attributed to eliminate the corresponding trap states present on the perovskite layer and reduce undesired non-radiative recombination.

To investigate the radiative recombination behavior and charge carrier dynamics of the perovskite films with and without EMB added, time-resolved photoluminescence (TRPL) measurements were carried out. These results is also corresponding to PL measurement, as shown in Figure 1c. TRPL decay traces were fitted with a bi-exponential decay model:

$I=A_{1} e^{-\frac{\mathrm{t}}{\tau_{1}}}+A_{2} \mathrm{e}^{-\frac{\mathrm{t}}{\tau_{2}}}$

in which I represents the normalized PL intensity, $A_{1}$ and $\mathrm{A}_{2}$ stand for the proportion of the components, and $\tau_{1}$ and $\tau_{2}$ represent the respective exciton lifetime for different carrier kinetic process. The average lifetime $\left(\tau_{\text {avg }}\right)$ is calculated in the following expression (2):

$\tau_{\text {avg }}=\frac{A_{1} \tau_{1}^{2}+A_{2} \tau_{2}^{2}}{A_{1} \tau_{1}+A_{2} \tau_{2}}$

The fitting results are shown in Table 2. the PL average lifetime of the quasi-2D perovskite films were increased from 52.07 to 70.34 ns after the $5 \mathrm{wt} \%$ EMB added, confirming the reduced possibility of defectinduced recombination. While $10 \mathrm{wt} \%$ EMB added and $15 \mathrm{wt} \%$ EMB added show a decrease in lifetime, which may be due to excessive ions destroying the microstructure of perovskite, resulting in the increase of trap states and the easier capture of excitons.

Table 2 PL lifetime obtained from fitted TRPL decay and average lifetime of the Perovskite film.

\begin{tabular}{|c|c|c|c|c|}
\hline & $\tau_{1}$ & $\tau_{2}$ & $\chi^{2}$ & $\tau_{\text {avg }}$ \\
\hline $\mathrm{w} / \mathrm{o}$ & 15.0459 & 80.1458 & 1.2603 & 52.0746 \\
\hline $5 \mathrm{w} \%$ & 23.3446 & 106.8600 & 1.0969 & 70.3441 \\
\hline $10 \mathrm{wt} \%$ & 19.6998 & 76.1083 & 1.1303 & 41.6401 \\
\hline $15 \mathrm{wt} \%$ & 14.5586 & 63.1357 & 1.0729 & 33.7271 \\
\hline
\end{tabular}


The morphology of the perovskite thin films was investigated by atomic force microscopy (AFM), as shown in Figure 2. The film without ionic liquid treatment shows an uneven gully shape, while the film with $5 \mathrm{wt} \% \mathrm{EMB}$ added is very smooth. It was obvious that the perovskite films showed more denser films without pinholes compared to the thin films without EMB treatment. We extracted root mean square roughness (RMS) from the AFM data. While the RMS of pristin film is about 20.3, which is much higher than that of $5 \mathrm{wt} \%$ EMB added-at about 3.27. This result shows that the ionic liquid we added can effectively improve the film quality of perovskite.
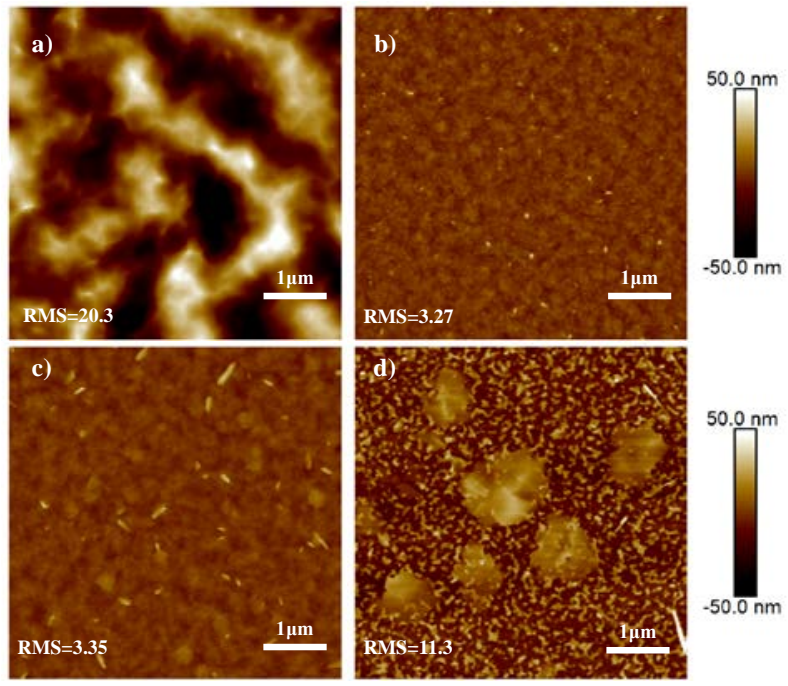

Figure 2 morphology characteristics. The atomic force microscopy of perovskite films of a)w/o EMB added b) $5 \mathrm{wt} \%$ EMB added c) 10 wt\% EMB added and d) 15 wt\% EMB added.

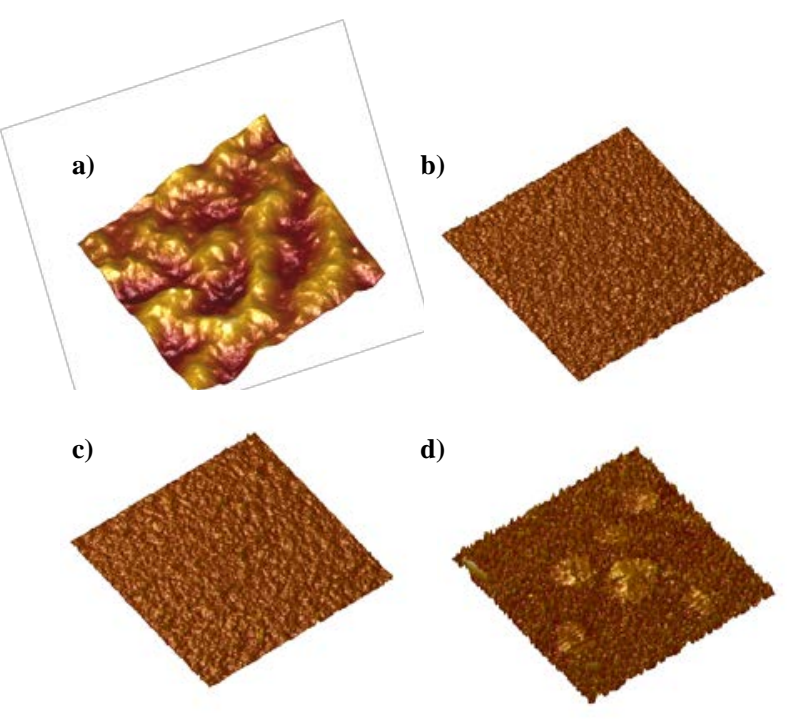

Figure 3 3D morphology characteristics. a)w/o EMB added b) $5 \mathrm{wt} \%$ EMB added c) $10 \mathrm{wt} \%$ EMB added and d) $15 \mathrm{wt} \%$ EMB added.
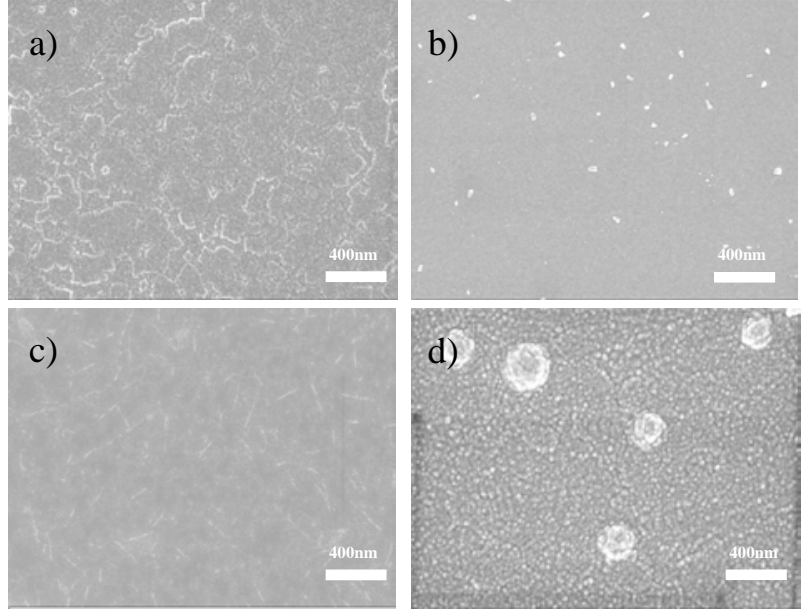

Figure 4 scanning electron microscopy (SEM) images of perovskite films of a)w/o EMB added b) 5 wt\% EMB added c) $10 \mathrm{wt} \%$ EMB added and d)15 wt\% EMB added.

Scanning electron microscopy (SEM) images of perovskite films with EMB and without EMB added were also carried out to further prove the improvement of film quality. The perovskite film that without EMB added exhibits imperfect surface coverage, resulting in very poor film morphology. After addition of $5 \mathrm{wt} \%$ EMB, the formed perovskite films show significant improvement in the surface coverage,and small pinholes were not observed. This corresponds well to the results obtained by AFM.

\section{Conclusion}

In summary, we demonstrate that EMB is an effective additive that can be utilized to gain control over the microstructure of perovskite thin films. At low concentrations, tuning the EMB content at $5 \mathrm{wt} \%$ allows us to get smooth thin films by over one order of magnitude.morever. The maximum photoluminescence intensity is increased by about 11 times, and the fluorescence lifetime is increased by about 1.5 times. Similarly, flat perovskite layers, as demonstrated in this work, can be of interest for application in other types of optoelectronic devices, such as LEDs.

\section{Acknowledgments}

This work was supported by the Natural Science Foundation of Jiangsu Province of China (Grant Nos. BK20190098, the Natural Science Foundation of the Jiangsu Higher Education Institutions of China (19KJA550001), Key Lab of Modern Optical Technologies of Education Ministry of China, Key Lab of Advanced Optical Manufacturing Technologies of Jiangsu Province, Priority Academic Program Development (PAPD) of Jiangsu Higher Education Institutions, and Jiangsu Shuangchuang Plan. 


\section{References}

1. Liang D, Peng Y, Fu Y, Shearer MJ, Zhang J, Zhai J, Zhang Y, Hamers RJ, Andrew TL, Jin S; 2016 Color-Pure Violet-Light-Emitting Diodes Based on Layered Lead Halide Perovskite Nanoplates. ACS Nano, 10:6897-6904.

2. Si J, Liu Y, He Z, Du H, Du K, Chen D, Li J, Xu M, Tian H, He H, et al; 2017 Efficient and High-ColorPurity Light-Emitting Diodes Based on In Situ Grown Films of CsPbX3 (X = Br, I) Nanoplates with Controlled Thicknesses. ACS Nano, 11:1110011107.

3. Byun J, Cho H, Wolf C, Jang M, Sadhanala A, Friend RH, Yang H, Lee TW; 2016 Efficient Visible Quasi-2D Perovskite Light-Emitting Diodes. Adv Mater, 28:7515-7520.

4. Song J, Li J, Xu L, Li J, Zhang F, Han B, Shan Q, Zeng H; 2018 Room-Temperature Triple-Ligand Surface Engineering Synergistically Boosts Ink Stability, Recombination Dynamics, and Charge Injection toward EQE-11.6\% Perovskite QLEDs. Adv Mater, 30:e1800764.

5. Liu Y, Yu Z, Chen S, Park JH, Jung ED, Lee S, Kang K, Ko S-J, Lim J, Song MH, et al; 2020 Boosting the Efficiency of Quasi-2D Perovskites Light-emitting Diodes by Using Encapsulation Growth Method. Nano Energy.

6. Han Y, Park S, Kim C, Lee M, Hwang I; 2019 Phase control of quasi-2D perovskites and improved light-emitting performance by excess organic cations and nanoparticle intercalation. Nanoscale, 11:3546-3556.

7. Jiang Y, Cui M, Li S, Sun C, Huang Y, Wei J, Zhang L, Lv M, Qin C, Liu Y, Yuan M; 2021 Reducing the impact of Auger recombination in quasi-2D perovskite light-emitting diodes. Nat Commun, 12:336.

8. Xiang C, Wu L, Lu Z, Li M, Wen Y, Yang Y, Liu W, Zhang T, Cao W, Tsang SW, et al; 2020 High efficiency and stability of ink-jet printed quantum dot light emitting diodes. Nat Commun, 11:1646.

9. Lin K, Xing J, Quan LN, de Arquer FPG, Gong X, Lu J, Xie L, Zhao W, Zhang D, Yan C, et al; 2018 Perovskite light-emitting diodes with external quantum efficiency exceeding 20 per cent. Nature, 562:245-248.

10. Liu XK, Xu W, Bai S, Jin Y, Wang J, Friend RH, Gao F; 2020 Metal halide perovskites for lightemitting diodes. Nat Mater.

11. Liang C, Gu H, Xia Y, Wang Z, Liu X, Xia J, Zuo S, Hu Y, Gao X, Hui W, et al; 2020 Two-dimensional Ruddlesden-Popper layered perovskite solar cells based on phase-pure thin films. Nature Energy.

12. Jang $\mathrm{CH}$, Harit AK, Lee S, Kim SH, Jeong JE, Park JH, Jung ED, Ha JM, Kwak SK, Woo HY, Song MH; 2020 Sky-Blue-Emissive Perovskite LightEmitting Diodes: Crystal Growth and Interfacial
Control Using Conjugated Polyelectrolytes as a Hole-Transporting Layer. ACS Nano.

13. Pang $P$, Jin $G$, Liang $C$, Wang $B$, Xiang W, Zhang D, $\mathrm{Xu} \mathrm{J}$, Hong W, Xiao Z, Wang L, et al; 2020 Rearranging Low-Dimensional Phase Distribution of Quasi-2D Perovskites for Efficient Sky-Blue Perovskite Light-Emitting Diodes. ACS Nano.

14. Lee S, Jang CH, Nguyen TL, Kim SH, Lee KM, Chang K, Choi SS, Kwak SK, Woo HY, Song MH; 2019 Conjugated Polyelectrolytes as Multifunctional Passivating and Hole-Transporting Layers for Efficient Perovskite Light-Emitting Diodes. Adv Mater, 31:e1900067. 